\title{
On equivariant mirror symmetry for local $\mathbb{P}^{2}$
}

\author{
Brian Forbes and Masao Jinzenji
}

\begin{abstract}
We solve the problem of equivariant mirror symmetry for $K_{\mathbb{P}^{2}}=$ $\mathcal{O}(-3) \rightarrow \mathbb{P}^{2}$ for the (three) cases of one independent equivariant parameter. This gives a decomposition of mirror symmetry for $K_{\mathbb{P}^{2}}$ into that of three subspaces, each of which may be considered independently. Finally, we give a new interpretation of mirror symmetry for $\mathcal{O}(k) \oplus \mathcal{O}(-2-k) \rightarrow \mathbb{P}^{1}$.
\end{abstract}

\section{Introduction}

\subsection{Background}

In recent years, our mathematical understanding of mirror symmetry has progressed dramatically (see in particular [4-8] for results relevant to the present paper). Nonetheless, there remains an entire theory of mirror symmetry which does not evidently fall under this umbrella, and which remains undeveloped; we shall call this as equivariant mirror symmetry.

Of course, as any theory of mirror symmetry must, our tentative 'equivariant mirror symmetry' has its roots in equivariant Gromov-Witten theory. What this means is essentially that, in addition to the usual data coming from the Gromov-Witten calculation, one must also keep track of the torus weights corresponding to the natural torus action on our (toric) Calabi-Yau space. The first example of this was detailed in [3], where they solved equivariant Gromov-Witten theory for arbitrary rank 2 bundles over a genus $g$ Riemann surface $\Sigma_{g}$, where one allows arbitrary torus weights $\left(\mu_{1}, \mu_{2}\right)$ to act on the bundle.

Using this example as a starting point, for the special case $\Sigma_{0}=\mathbb{P}^{1}$, we sucessfully worked out the equivariant mirror computation in $[5,6]$. Namely, it was shown in [5] that by appropriately adapting the results of [4], the genus zero invariants of [3] could be computed via mirror symmetry. This approach was simplified and streamlined in [6], where a formula for the mirror map agreeing with the physical analysis of [10] was derived (as well as Picard-Fuchs equations and Yukawa couplings). 
The above then naturally leads us to the question of how to describe equivariant mirror symmetry for the next most complicated example: $K_{\mathbb{P}^{2}}=$ $\mathcal{O}(-3) \rightarrow \mathbb{P}^{2}$. Although the only difference between this and the examples of $[5,6]$ is that now $b_{4}\left(K_{\mathbb{P}^{2}}\right) \neq 0$, as we will see below, even this small difference leads to a vastly more challenging problem. Nonetheless, we find that an approach which is more or less the natural generalization of $[5,6]$ allows us to deal with the mirror symmetry problem for this space, in the case that we have only one independent equivariant parameter. We check our results via the (Chern-Simons) computations of [1] and find agreement with their results. As the Chern-Simons theory contains significantly more data than ordinary mirror symmetry, our calculations represent a substantial refinement of mirror symmetry. Remarkably, we also find a method of modifying the usual localization calculation for $K_{\mathbb{P}^{2}}$ which exactly reproduces the 'refined Gromov-Witten invariants' of [1].

In addition, we find a new and simplified approach to deriving the results of [6], which allows us to produce closed formulas for the two-curve formula (defined below).

The organization of this paper is as follows. Section 2 briefly outlines the approach of [1] to the equivariant mirror problem. Section 3 establishes the equivariant mirror calculation for $K_{\mathbb{P}^{2}}$ for one independent equivariant parameter (this gives three separate cases; see figure 1) both by directly using

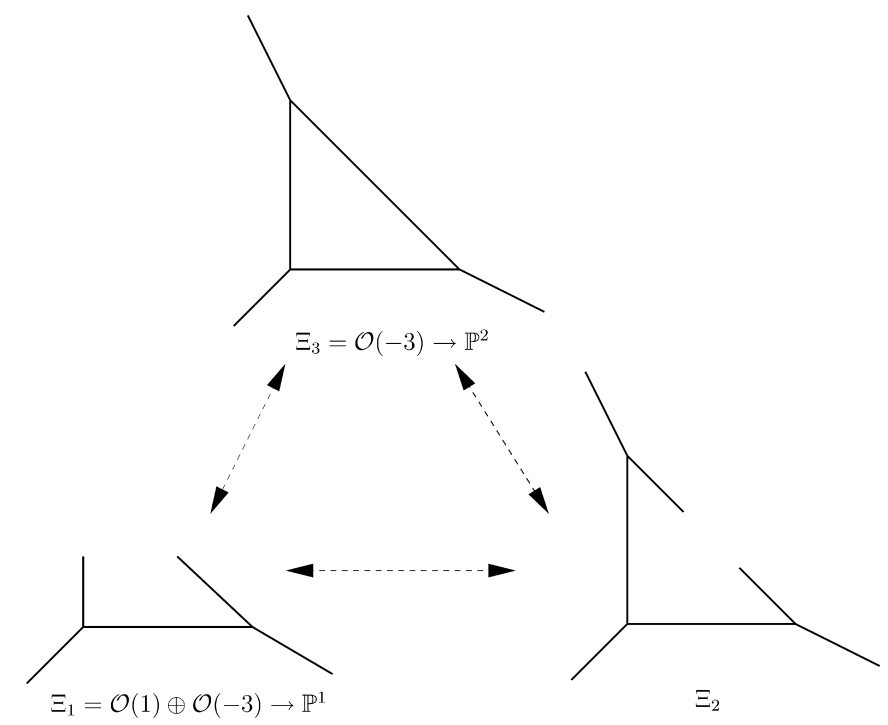

Figure 1: Decomposition of $K_{\mathbb{P}^{2}}$ via equivariant parameters. 
[5] and by applying the simplifications of [6]. Section 4 gives a novel approach to localization which allows us to directly work out the fully equivariant Gromov-Witten theory of $K_{\mathbb{P}^{2}}$. Finally, Section 5 presents our new approach to the problem of $\mathcal{O}(k) \oplus \mathcal{O}(-2-k) \rightarrow \mathbb{P}^{1}$. Relevant facts about $I$ and $J$ functions and their relation to mirror symmetry is presented in the appendix.

\subsection{Summary of results}

In this paper, we work out equivariant mirror symmetry for $\mathcal{O}(-3) \rightarrow \mathbb{P}^{2}$, for the case of one independent equivariant parameter. The geometric meaning of this is depicted in figure 1: certain choices of equivariant weights decompose $\mathcal{O}(-3) \rightarrow \mathbb{P}^{2}$ into a family of three subspaces $\Xi_{1}=\mathcal{O}(1) \oplus \mathcal{O}(-3) \rightarrow$ $\mathbb{P}^{1}, \Xi_{2}$ and $\Xi_{3}=\mathcal{O}(-3) \rightarrow \mathbb{P}^{2}$, and we give mirror symmetry constructions which reproduce the Gromov-Witten invariants of each.

Concretely, we find the following:

Proposition 1.1. Mirror symmetry for the spaces $\Xi_{1}, \Xi_{2}$ and $\Xi_{3}$ is the same as that of

$$
\begin{aligned}
& \Xi_{1}: \mathcal{O}(1)_{\nu} \oplus \mathcal{O}(-3)_{-\nu} \longrightarrow \mathbb{P}_{[0,0]}^{1} \\
& \Xi_{2}: \mathcal{O}(1)_{\nu} \oplus \mathcal{O}(-3)_{-2 \nu} \longrightarrow \mathbb{P}_{[0, \nu]}^{1} \\
& \Xi_{3}: \mathcal{O}(-3)_{-3 \nu} \rightarrow \mathbb{P}_{[\nu, \nu, \nu]}^{2}
\end{aligned}
$$

where the subscripts denote equivariant weights acting on each term. Equivalently, this can be represented by the following toric data subject to the cohomology relations:

$$
\begin{aligned}
& \left(\begin{array}{llll}
1 & 1 & 1 & -3 \\
& & \nu & -\nu
\end{array}\right): p^{2}=0 \\
& \left(\begin{array}{cccc}
1 & 1 & 1 & -3 \\
& \nu & \nu & -2 \nu
\end{array}\right): p(p+\nu)=0 \\
& \left(\begin{array}{cccc}
1 & 1 & 1 & -3 \\
\nu & \nu & \nu & -3 \nu
\end{array}\right):(p+\nu)^{3}=0
\end{aligned}
$$

Above, the second row of each matrix specifies the equivariant weights, and $\left(\begin{array}{llll}1 & 1 & 1 & -3\end{array}\right)$ is the standard toric vector for $\mathcal{O}(-3) \rightarrow \mathbb{P}^{2}$.

We use this to confirm a prediction of [1] regarding the relationship of the so-called 'refined Gromov-Witten invariants' computed via Chern-Simons theory in that paper, and equivariant Gromov-Witten invariants. 
We expect that the eventual 'full' equivariant mirror symmetry computation (which will give a Gromov-Witten generating function agreeing with [1]) will use our methods to some extent. However, at present, the complicated interplay of Birkhoff factorized and non-Birkhoff factorized functions involved have put the full theory out of reach.

The second result of this paper is a new interpretation of the mirror symmetry calculation of $\mathcal{O}(k) \oplus \mathcal{O}(-2-k) \rightarrow \mathbb{P}^{1}$ with antidiagonal equivariant weights $(\nu,-\nu)$ acting on the bundle. We find

Proposition 1.2. Mirror symmetry for $X_{k}=\mathcal{O}(k) \oplus \mathcal{O}(-2-k) \rightarrow \mathbb{P}^{1}$ with the antidiagonal action (the equivariantly Calabi-Yau case) can be determined from the toric data

$$
\left(\begin{array}{cccccc}
1 & 1 & 1 & -1 & -1 & -1 \\
& & \nu / k & -\nu / k & -\nu & -\nu
\end{array}\right)
$$

which corresponds to the geometry

$$
\mathcal{O}(1)_{\nu / k} \oplus \mathcal{O}(-1)_{-\nu / k} \oplus \mathcal{O}(-1)_{-\nu} \oplus \mathcal{O}(-1)_{-\nu} \rightarrow \mathbb{P}^{1}
$$

where the subscripts denote equivariant weights (and we are considering the trivial action on $\left.\mathbb{P}^{1}\right)$. This reproduces the mirror maps of $[6,10]$ and gives a closed formula for the generating function of Gromov-Witten invariants of $X_{k}$ :

$$
W_{k}(x)=\sum_{j=1}^{\infty} \frac{(-1)^{(j-1) k}}{j^{2}(j-1) !} \prod_{m=1}^{j-1}(k(2+k) j+m) x^{k}
$$

Finally, we give a generalization of the above result which gives closed formulas for the mirror map and Gromov-Witten invariants of a large class of geometries.

\section{The idea of equivariant mirror symmetry}

Here, we would like to outline the general principles that guide our study of equivariant mirror symmetry.

We recall [1, Section 7.6] and its application to our current problem, $K_{\mathbb{P}^{2}}$. The idea there was to define a set of 'refined' Gromov-Witten invariants of $K_{\mathbb{P}^{2}}$ by setting the Kähler parameter of each $\mathbb{P}^{1} \hookrightarrow \mathbb{P}^{2}$ to a different value, which is apparently a natural operation from the vantage of the dual ChernSimons theory. They then went on to compute these new Gromov-Witten 
invariants as rational functions of the three Kähler parameters. The expectation is that these should somehow correspond to the equivariant GromovWitten theory of $K_{\mathbb{P}^{2}}$, with equivariant weights given as (M. Marino, private communication):

$$
\left(\begin{array}{cccc}
1 & 1 & 1 & -3 \\
\mu_{1} & \mu_{2} & \mu_{3} & -\mu_{1}-\mu_{2}-\mu_{3}
\end{array}\right) .
$$

While these two concepts are not readily seen to be the same, in this paper, for the cases $\left(\mu_{1}, \mu_{2}, \mu_{3}\right)=(0,0, \nu),(0, \nu, \nu)$ and $(\nu, \nu, \nu)$, we find agreement with the results of [1].

A general formulation of what is being computed in [1] would go something as follows. Let $X$ be a non-compact toric Calabi-Yau 3-fold, and let $\Gamma$ be the image of the moment map, i.e., the toric graph associated to $X$. We denote the set of bounded edges of $\Gamma$ by $\left\{\gamma_{i}\right\}$, and associate a parameter $\nu_{i}$ to each $\gamma_{i}$. Then the generating function of Gromov-Witten invariants of [1] will be a function

$$
W\left(\nu_{1} \cdots \nu_{n}\right) \in \mathbb{Q}\left[\nu_{1} \cdots \nu_{n}\right][[x]]
$$

such that e.g., $W\left(\nu_{1}, \nu_{2}=0 \cdots \nu_{n}=0\right)$ is the generating function of GromovWitten invariants of maps

$$
f: \mathbb{P}^{1} \rightarrow X, \quad f_{*}\left(\mathbb{P}^{1}\right) \in \gamma_{1} .
$$

Similarly, $W\left(\nu_{1}, \nu_{2}, \nu_{3}=0 \cdots \nu_{n}=0\right)$ will count maps from $\mathbb{P}^{1}$ into $\gamma_{1} \cup \gamma_{2}$, etc. Then mirror symmetry in this sense would mean that there would be a mirror manifold $Y_{\nu_{1} \cdots \nu_{n}}$ to $X$ such that the period integrals of $Y$ would allow us to compute $W\left(\nu_{1} \cdots \nu_{n}\right)$. According to this picture, in addition to ordinary mirror symmetry, equivariant mirror symmetry should also include mirror symmetry for each

$$
\left(N_{C / X} \rightarrow C\right) \hookrightarrow X
$$

for each $C \hookrightarrow X$, and for each pair of curves in $X$, etc. Moreover, all of these should be incorporated into a single picture with the use of the parameters $\nu_{i}$.

\section{Equivariant mirror symmetry for $K_{\mathbb{P}^{2}}$ : one independent equivariant parameter}

We now present the techniques for the equivariant mirror symmetry calculation corresponding to figure 1 . The methods are surprisingly simple, 
yet require some highly non-trivial observations in order to be successfully carried out.

\subsection{Review of $\Xi_{1}=\mathcal{O}(1) \oplus \mathcal{O}(-3) \rightarrow \mathbb{P}^{1}$}

We first review the content of [5], which established the mirror symmetry calculation for $\Xi_{1}=\mathcal{O}(1) \oplus \mathcal{O}(-3) \rightarrow \mathbb{P}^{1}$. Recall that the toric data describing $K_{\mathbb{P}^{2}}$ is just

$$
\left(\begin{array}{llll}
1 & 1 & 1 & -3
\end{array}\right)
$$

which is a shorthand notation for the toric quotient

$$
K_{\mathbb{P}^{2}}=\left\{\left(z_{i}\right) \in \mathbb{C}^{4}:\left|z_{1}\right|^{2}+\left|z_{2}\right|^{2}+\left|z_{3}\right|^{2}-3\left|z_{4}\right|^{2}=r\right\} / S^{1}
$$

where $r \in \mathbb{R}^{+}$and

$$
S^{1}:\left(z_{1} \cdots z_{4}\right) \longrightarrow\left(e^{i \theta} z_{1}, e^{i \theta} z_{2}, e^{i \theta} z_{3}, e^{-3 i \theta} z_{4}\right)
$$

From this, we see immediately that the three 1s in (3.1) represent homogeneous coordinates on $\mathbb{P}^{2}$. So, in order to get at the geometry of $\mathcal{O}(1) \oplus$ $\mathcal{O}(-3) \rightarrow \mathbb{P}^{1}$, we want to treat $z_{3}$ as a fiber variable with respect to $\mathbb{P}^{1}=$ $\left[z_{1}, z_{2}\right]$. This can be accomplished by considering a partially equivariant theory on $K_{\mathbb{P}^{2}}$ :

$$
\left(\begin{array}{llll}
1 & 1 & 1 & -3 \\
& & \nu & -\nu
\end{array}\right)
$$

where $\nu \in \mathbb{C}^{*}$ and the second column represents the torus weights acting on the coordinates of $K_{\mathbb{P}^{2}}$. As explained below, we have to subject the $I$ function corresponding to this vector to the cohomology relation $p^{2}=0$, which implies that we are considering the geometry

$$
\mathcal{O}(1)_{\nu} \oplus \mathcal{O}(-3)_{-\nu} \rightarrow \mathbb{P}_{[0,0]}^{1}
$$

Then, by expanding in $\nu=\infty$ and performing Birkhoff factorization, as advocated in [4], we can indeed realize mirror symmetry for $\mathcal{O}(1) \oplus \mathcal{O}(-3) \rightarrow \mathbb{P}^{1}$. 
Concretely, this goes as follows. The $I$ function corresponding to (3.4) is a cohomology-valued hypergeometric series

$$
I_{1}=q^{p / \hbar} \sum_{d \geq 0} \frac{\prod_{m=-3 d+1}^{0}(-3 p-\nu+m \hbar)}{\prod_{m=1}^{d}(p+\nu+m \hbar) \prod_{m=1}^{d}(p+m \hbar)^{2}} q^{d},
$$

which is annihilated by the equivariant Picard-Fuchs operator

$$
\begin{aligned}
\mathcal{D}_{1} & =\theta^{2}(\theta+\nu)-q(-3 \theta-\nu)(-3 \theta-\nu-\hbar)(-3 \theta-\nu-2 \hbar), \\
\theta & =\hbar q \frac{d}{d q}
\end{aligned}
$$

Above, $p$ is the Kähler class on $\mathbb{P}^{1}$, which means that we enforce the cohomology relation $p^{2}=0$ in $I$. This is equivalent to considering just two of the three solutions of $\mathcal{D}_{1} f=0$ :

$$
\left.I\right|_{p=0},\left.\quad \frac{d}{d p} I\right|_{p=0} .
$$

We then Birkhoff factorize these two to recover a $J$ function, and subsequently transform by the mirror map, which gives us the instanton expansion for the $\Xi_{1}$ geometry:

$$
\begin{aligned}
W_{\Xi_{1}} & =\sum_{k>0} \frac{(-1)^{k-1}}{k^{2}(k-1) !} \prod_{j=1}^{k-1}(3 k+j) x^{k} \\
& =x-\frac{7}{4} x^{2}+\frac{55}{9} x^{3}-\frac{455}{16} x^{4}+\frac{3876}{25} x^{5}-\frac{33649}{36} x^{6}+\cdots
\end{aligned}
$$

Note that this is the derivative of the prepotential of $\Xi_{1}=\mathcal{O}(1) \oplus \mathcal{O}(-3) \rightarrow$ $\mathbb{P}^{1}$, which must be (logarithmically) integrated once in $x$ to compute integer invariants via the multiple cover formula.

\section{2. $\Xi_{2}$ : Two $(1,-3)$ curves in $K_{\mathbb{P}^{2}}$}

Before diving into the details, we make some effort at clarifying the meaning of the space $\Xi_{2}$, which is represented in figure 2 . The basic intuition is that we should consider the configuration of two curves, intersecting at right angles, such that the normal bundle of each is $\mathcal{O}(1) \oplus \mathcal{O}(-3) \rightarrow \mathbb{P}^{1}$, and also such that the $\mathcal{O}(-3)$ factor of the two curves is the same.

Unfortunately, at present, we lack a rigorous mathematical definition for $\Xi_{2}$. However, when viewed as a localization problem, the idea behind it is 


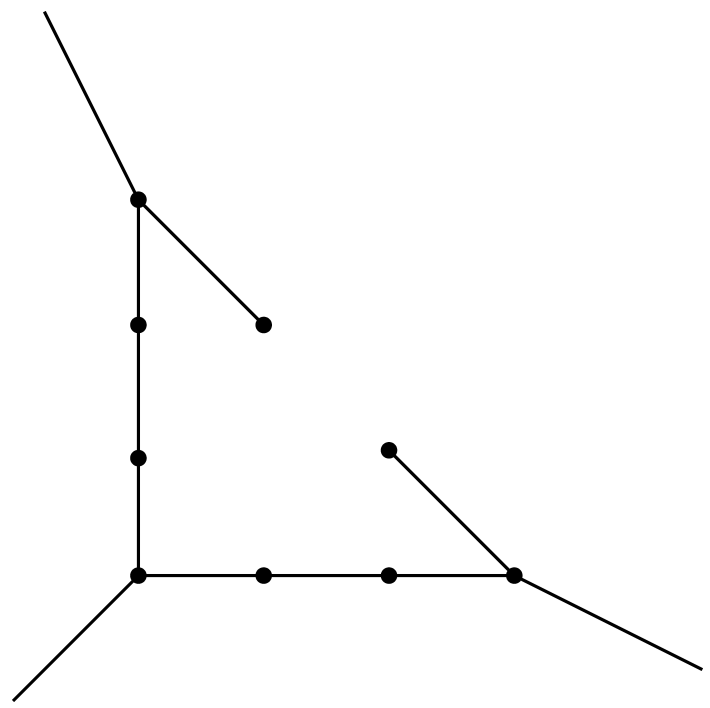

Figure 2: The two-curve problem $\Xi_{2}$.

simple. Ordinary Gromov-Witten theory of $K_{\mathbb{P}^{2}}$ computes some number based on the moduli space of degree $d$ maps

$$
N_{d}=\#\left\{f: \mathbb{P}^{1} \rightarrow K_{\mathbb{P}^{2}}\right\} .
$$

Now, in order to define the Gromov-Witten invariants of $\Xi_{2}$, we just have to remove certain contributions from this number. Namely, if $C_{i}, i=1,2,3$ represent the three $\mathbb{P}^{1} \mathrm{~s}$ in $\mathbb{P}^{2}$, then we want to remove all curves which contain a component mapping to $C_{3}$.

From the physics point of view, the dual Chern-Simons theory evidently provides some sort of definition for $\Xi_{2}[1]$, since [1] was able to produce Gromov-Witten invariants for $\Xi_{2}$. However, this relies on large $N$ duality, which has clearly not been proven mathematically. Another possibility, though equally unproven, would be to define this space by its equivalence to the theory of open strings with two Lagrangian submanifolds on $\mathbb{C}^{3}$ (briefly outlined later in the paper). Note that this is equivalent to the two-leg topological vertex, with appropriately chosen framings.

At any rate, even in absence of a rigorous definition of $\Xi_{2}$, the space does have known Gromov-Witten invariants, and hence we can approach the problem by simply looking for a toric model which reproduces these invariants through mirror symmetry. We use the above intuitive picture of $\Xi_{2}$ as a guide in seeking the correct toric data. Remarkably, we find that the 
most obvious choice of (equivariant) toric vector precisely describes mirror symmetry for $\Xi_{2}$ (though some highly non-trivial manipulations are required to demonstrate this).

The Gromov-Witten invariants of $\Xi_{2}$ can be computed from the following generating function, which is the derivative of the prepotential for the two-curve case $\Xi_{2}$ (note the dramatic similarity with the $\mathcal{O}(1) \oplus \mathcal{O}(-3) \rightarrow$ $\mathbb{P}^{1}$ prepotential of equation $\left.(3.9)\right)$ :

$$
\begin{aligned}
W_{\Xi_{2}}= & 2 \sum_{k>0} \frac{(-1)^{k-1}}{k^{2}(k-1) !} \prod_{j=1}^{k-1}(5 k+j) x_{1}^{k} \\
= & 2 x_{1}-\frac{11}{2} x_{1}^{2}+\frac{272}{9} x_{1}^{3}-\frac{1771}{8} x_{1}^{4} \\
& +\frac{47502}{25} x_{1}^{5}-\frac{162316}{9} x_{1}^{6}+\cdots
\end{aligned}
$$

Note that this is a function of only one Kähler parameter. In order to see a two Kähler parameter expansion, one would have to use two different equivariant parameters; however, incorporating this into the full equivariant mirror calculation of $K_{\mathbb{P}^{2}}$ is beyond the scope of the present work.

So, on to the calculation. From the previous section, we saw that the $\Xi_{1}$ calculation could be done by use of the toric data

$$
\left(\begin{array}{llll}
1 & 1 & 1 & -3 \\
& & \nu & -\nu
\end{array}\right)
$$

together with some semi-involved manipulations of the period integrals $[4,5]$. Thus, the choice which immediately springs to mind for use on $\Xi_{2}$ is

$$
\left(\begin{array}{cccc}
1 & 1 & 1 & -3 \\
& \nu & \nu & -2 \nu
\end{array}\right)
$$

for mirror symmetry. However, there are major problems with this that make it seem far too naive to be the right toric data. Namely, on $\Xi_{1}=$ $\mathcal{O}(1) \oplus \mathcal{O}(-3) \rightarrow \mathbb{P}^{1}$ above, we could apply the cohomology relation $p^{2}=0$ for $\mathbb{P}^{1}$ to the $I$ function. This relation then dictates that we must expand the $I$ function in $\nu=\infty$ [4], and we can then use Birkhoff factorization to exhibit mirror symmetry. However, on $\Xi_{2}$, since we cannot even define the space mathematically, we have no guess as to what cohomology relation to use, and therefore no way of knowing the correct asymptotic expansion of $\nu$.

Nonetheless, we will show here that one basic assumption allows us to essentially use (3.14) to exhibit mirror symmetry for $\Xi_{2}$. The assumption is, 
we only need to use two of the three solutions of the associated equivariant Picard-Fuchs system in order to determine mirror symmetry. The justification for this assumption is that, on both $\mathcal{O}(1) \oplus \mathcal{O}(-3) \rightarrow \mathbb{P}^{1}$ and (equivariant) $\mathcal{O}(-3) \rightarrow \mathbb{P}^{2}$, computation of the prepotential requires only two of three Picard-Fuchs solutions. Thus, it is reasonable to hope that the 'intermediate' case $\Xi_{2}$ also enjoys this feature.

We can now see relatively simply that this assumption allows us to derive mirror symmetry for $\Xi_{2}$. The first implication of our assumption is that we have to work instead with an equivariant theory given by

$$
\left(\begin{array}{cccc}
1 & 1 & 1 & -3 \\
& \mu & \nu & -2 \nu
\end{array}\right)
$$

The reason for using a new parameter $\mu$ is as follows. The Picard-Fuchs system associated to (3.15) is

$$
\mathcal{D}_{2}=\theta(\theta+\mu)(\theta+\nu)+q(3 \theta+2 \nu)(3 \theta+2 \nu+\hbar)(3 \theta+2 \nu+2 \hbar) .
$$

Then if we set

$$
I_{2}=q^{p / \hbar} \sum_{d \geq 0} \frac{\prod_{m=-3 d+1}^{0}(-3 p-2 \nu+m \hbar)}{\prod_{m=1}^{d}(p+\mu+m \hbar) \prod_{m=1}^{d}(p+\nu+m \hbar) \prod_{m=1}^{d}(p+m \hbar)} q^{d},
$$

the three solutions of $\mathcal{D}_{2} f=0$ are given by

$$
\left.I_{2}\right|_{p=0},\left.\quad I_{2}\right|_{p=-\mu},\left.\quad I_{2}\right|_{p=-\nu} .
$$

Under our assumption, we want to use two of these three solutions for our mirror symmetry calculation. If we use the first two of these solutions, this will force us to asymptotically expand $I_{2}$ in $\mu=0, \nu=\infty$. This is because, using only the first two solutions is equivalent to subjecting $I_{2}$ to the cohomology relation $p(p+\mu)=0$, which forces a $\mu=0$ expansion. This then implies that the variables involving $\nu$ are 'fiber' variables with respect to the equivariant $\mathbb{P}^{1}$ given by $p(p+\mu)=0$, and hence must be expanded in $\nu=\infty$, according to [4].

So, in summary, since our assumption requires that $I_{2}$ be expanded in $\mu=0, \nu=\infty$, we see the necessity of introducing a new equivariant parameter $\mu$ compared to our original guess (3.14). 
We briefly note that the geometry (3.15) subject to the cohomology relation $p(p+\mu)=0$ is given by

$$
\mathcal{O}(1)_{\nu} \oplus \mathcal{O}(-3)_{-2 \nu} \rightarrow \mathbb{P}_{[0, \mu]}^{1}
$$

Now that we have fully set up the problem, we have only to carry out the calculation. We expand $I_{2}$ as

$$
\begin{aligned}
& \frac{1}{\prod_{m=1}^{d}(p+\nu+m \hbar)}, \quad \text { in } \hbar=0 \\
& \frac{1}{\prod_{m=1}^{d}(p+\mu+m \hbar)}, \quad \text { in } \hbar=\infty .
\end{aligned}
$$

By using the equality

$$
\left.\mu \frac{d}{d p} I_{2}\right|_{p=0}=-\left.I_{2}\right|_{p=0}+\left.I_{2}\right|_{p=-\mu},
$$

we can perform Birkhoff factorization of the two solutions $\left.I_{2}\right|_{p=0}$, $\left.(d / d p) I_{2}\right|_{p=0}$. The result is a $J$ function

$$
J_{\mu, \nu=1}=1+\frac{p t+t_{0}}{\hbar}+\frac{((3 \mu-5) p+2) W}{\hbar^{2}}+\cdots
$$

where the mirror map $t$ has a form which is unfortunately not defined in the $\mu \rightarrow 1$ limit (we have set $\nu=1$ for simplicity):

$$
\begin{aligned}
t= & -\left(5+6 \mu+(\mu-1)^{-1}\right) q \\
& +\left(-\frac{33}{2}+117 \mu-\frac{1}{2}\left[(\mu-1)^{-1}+7(\mu-1)^{-2}+(\mu-1)^{-3}\right]\right) q^{2}+\cdots
\end{aligned}
$$

Remarkably, $W$ does turn out to be well defined for $\mu=1$ :

$$
\begin{aligned}
W_{\mu, \nu=1}(x)= & (2 \mu-4) x+\left(26-\frac{53}{2} \mu+6 \mu^{2}\right) x^{2} \\
& -\left(\frac{3028}{9}-\frac{4646}{9} \mu+246 \mu^{2}-36 \mu^{3}\right) x^{3} \\
& +\left(\frac{11601}{2}-\frac{95077}{8} \mu+\frac{17363}{2} \mu^{2}-2664 \mu^{3}+288 \mu^{4}\right) x^{4}+\cdots
\end{aligned}
$$


where $x=e^{t}$. Then, simply setting $\mu=1$ gives the two-curve expansion (3.11).

$$
W_{\mu=1, \nu=1}(x)=-2 x+\frac{11}{2} x^{2}-\frac{272}{9} x^{3}+\frac{1771}{8} x^{4}+\cdots .
$$

Hence, we have accomplished our aim of exhibiting mirror symmetry for the space $\Xi_{2}$.

We also note that we can perform another consistency check of this calculation, which gives geometric insight into why this method produces invariants for $\Xi_{2}$. That is, by setting $\mu=0$ in $W_{\mu, \nu=1}(x)$, the resulting invariants agree with those of

$$
\mathcal{O}(1)_{\nu} \oplus \mathcal{O}(-3)_{-2 \nu} \rightarrow \mathbb{P}_{[0,0]}^{1}
$$

Of course, this is precisely the $I$ function we get by setting $\mu=0$ into $I_{2}$, so this is consistent. What this also says is that the $\mu$ parameter is somehow encoding the information of an extra divisor in $K_{\mathbb{P}^{2}}$, i.e., without $\mu$, the result collapses into the invariants of a single $\mathbb{P}^{1} \hookrightarrow K_{\mathbb{P}^{2}}$.

As a final note, we mention that this computation can clearly be combined with the $\mathcal{O}(1) \oplus \mathcal{O}(-3) \rightarrow \mathbb{P}^{1}$ calculation of the previous section (and also with the full $K_{\mathbb{P}^{2}}$ case, in fact) simply by using the toric vector

$$
\left(\begin{array}{cccc}
1 & 1 & 1 & -3 \\
& k \mu & \nu & -(k+1) \nu
\end{array}\right): p(p+k \mu)=0
$$

and going through the same steps as above. Then $k=0$ will give $\Xi_{1}$ and $k=1$ gives $\Xi_{2}$.

\section{3. $\Xi_{3}=K_{\mathbb{P}^{2}}$ with equivariant parameters}

Finally, we give a brief discussion on including $K_{\mathbb{P}^{2}}$ into the above picture. Although this is the only of the three spaces for which standard mirror symmetry actually applies, how to incorporate this into the above Birkhoff factorization scheme is not immediately clear. This is because, as we will see, a Birkhoff factorized function cannot be used to compute $K_{\mathbb{P}^{2}}$ invariants, which necessitates including both the factorized and unfactorized functions together. Nonetheless, certain coincidences in the Birkhoff factorization make this possible. 
Now, in analogy with (3.14) above, the natural thing to use is

$$
\left(\begin{array}{cccc}
1 & 1 & 1 & -3 \\
\nu & \nu & \nu & -3 \nu
\end{array}\right),
$$

and the most naive application of the above machinery would be to try to work with

$$
\left(\begin{array}{cccc}
1 & 1 & 1 & -3 \\
\mu & \mu & \nu & -3 \nu
\end{array}\right),
$$

with $\mu=0, \nu=\infty$. However, we quickly see that this can not be right, since the computation reveals that the Gromov-Witten invariants here are the same as those of $\mathcal{O}(1)_{\nu} \oplus \mathcal{O}(-3)_{-3 \nu} \rightarrow \mathbb{P}_{[0,0]}^{1}$. Further investigation shows that the prepotential $\mathcal{F}$ of the most general configuration $\mathcal{O}(1)_{\nu_{1}} \oplus$ $\mathcal{O}(-3)_{\nu_{2}} \rightarrow \mathbb{P}_{\left[\mu_{1}, \mu_{2}\right]}^{1}$ is a function of only two parameters:

$$
\mathcal{F}=\mathcal{F}\left(\mu_{1}-\mu_{2}, \frac{\nu_{2}}{\nu_{1}}\right) .
$$

It is not hard to show that the invariants of $K_{\mathbb{P}^{2}}$ are not included here. This might have been guessed at, as the Birkhoff factorization necessarily removes some information from the fundamental solution of $K_{\mathbb{P}^{2}}$, and we need all the data of the original function to compute the right invariants.

Hence, we have to pursue another approach. As we now show, a simple trick actually allows us to simultaneously express the invariants corresponding to the $\hbar$ and $1 / \hbar$ expansion of the $I$ function.

For simplicity, we consider only a single parameter in equivariant cohomology, i.e., $H_{\mathbb{C}^{*}}^{*}\left(\mathbb{P}^{2}\right)$. (Note that, by (3.30), this is equivalent to using (3.29) if we change $-3 \nu$ to $-k \nu, k \in \mathbb{Z}$.) This is the same setup we had in Section 3.1:

$$
\left(\begin{array}{llll}
1 & 1 & 1 & -3 \\
& & \nu & -\nu
\end{array}\right),
$$

but the interpretation will be a bit different this time. First, one can compute readily that, when we expand the $I$ function corresponding to this vector, $I_{1}$, in $\hbar=\infty$, we can see the $K_{\mathbb{P}^{2}}$ Gromov-Witten invariants (even after imposing the $p^{2}=0$ relation). This is just due to the fact that the $I$ functions for $K_{\mathbb{P}^{2}}$ and the $(1,-3)$ curve are the same. Then, as the $\mathcal{O}(1) \oplus \mathcal{O}(-3) \rightarrow \mathbb{P}^{1}$ invariants were also computed using $p^{2}=0$, this is simply a matter of putting both pieces together. 
Ultimately, the connection between the $K_{\mathbb{P}^{2}}$ calculation and those of the previous two sections is that the Birkhoff factorization takes on a special form in this case. Recall that Birkhoff factorization applied to the fundamental solution $S$ (see the appendix) produces

$$
S\left(\hbar, \hbar^{-1}\right)=Q^{-1}(\hbar) R\left(\hbar^{-1}\right)
$$

From what we mentioned above, in our case the fundamental solution can be taken as

$$
S^{t}=\left(\left.\left.I\right|_{p=0} \quad \frac{d}{d p} I\right|_{p=0}\right)
$$

Now, the Birkhoff factorization (3.32) can be rewritten more precisely as

$$
S\left(q, \hbar, \hbar^{-1}\right)=\left(I_{2}+\sum_{k>0} Q_{k} q^{k}\right)^{-1} R\left(q, \hbar^{-1}\right)
$$

where $I_{2}$ is the 2-dimensional identity matrix and we have set

$$
A=\sum_{k \geq 0} A_{k} q^{k}
$$

for a matrix $A$. Looking back at the $I$ function $I_{1}$ of Subsection 3.1, it is evident that in the expansion $\nu=\infty$, the only term contributing positive powers of $\hbar$ to $I_{1}$ is

$$
\frac{1}{\prod_{m=1}^{d}(p+\nu+m \hbar)} .
$$

This implies that the $Q$ matrix of the Birkhoff factorization for this case takes on the following special form:

$$
Q_{k}=Q_{k}\left((\hbar+\nu)^{-1},(2 \hbar+\nu)^{-1}, \ldots,(k \hbar+\nu)^{-1}\right) .
$$

In other words, $Q$ makes sense when expanded as a power series in either $\hbar$ or $1 / \hbar$ !

We can use this to immediately write down a function which posesses $\Xi_{3}=K_{\mathbb{P}^{2}}$ and $\Xi_{1}=\mathcal{O}(1) \oplus \mathcal{O}(-3) \rightarrow \mathbb{P}^{1}$ invariants. (Note that this can readily be modified to include $\Xi_{2}$ invariants as well, via the comment at the end of Section 3.2.) This is more than a bit surprising, as $\Xi_{1}$ requires Birkhoff factorization while $\Xi_{3}$ does not, i.e., the asymptotic expansion of 
$\nu$ for each respective space appears incompatible. At any rate, noting our well-behaved $Q$ above, we simply modify the factorization by inserting a parameter $w$ :

$$
S^{\prime}\left(q, \hbar, \hbar^{-1}, w\right)=\left(I_{2}+w \sum_{k>0} Q_{k} q^{k}\right)^{-1} R\left(q, \hbar^{-1}\right)
$$

We now treat $S^{\prime}$ as a function of $1 / \hbar$ and expand. The result is a new $I$ function

$$
I^{\prime}=1+\frac{p t+t_{0}}{\hbar}+\frac{W}{\hbar^{2}}+\cdots
$$

where everything is now $w$ dependent. The mirror maps are

$$
\begin{aligned}
t_{0}= & -2 q_{1}+(17-2 w) q_{1}^{2}+\left(50 w-\frac{710}{3}\right) q_{1}^{3} \\
& +\left(\frac{8049}{2}-1137 w\right) q_{1}^{4}+\cdots \\
t= & (2 w-8) q_{1}+(74-29 w) q_{1}^{2}+\left(\frac{1532}{3} w-\frac{3212}{3}\right) q_{1}^{3} \\
& +\left(18609-\frac{19893}{2} w\right) q_{1}^{4}+\cdots
\end{aligned}
$$

from which we can easily make a consistency check, in that $w=0$ gives the mirror map for $\Xi_{1}$ while $w=1$ is the mirror map for $K_{\mathbb{P}^{2}}$. After inverting this and changing variables, we find

$$
\begin{aligned}
\left.W\right|_{p=0}= & x_{1}-\left(2 w+\frac{7}{4}\right) x_{1}^{2}+\left(6 w^{2}+15 w+\frac{55}{9}\right) x_{1}^{3} \\
& -\left(\frac{64}{3} w^{3}+100 w^{2}+\frac{643}{6} w+\frac{455}{16}\right) x_{1}^{4}+\cdots
\end{aligned}
$$

where we have set $p=0$ to avoid dealing with the leading Kähler parameter factor. ${ }^{1}$ Then one sees immediately that $w=0$ gives $\Xi_{1}$ invariants while $w=1$ gives $K_{\mathbb{P}^{2}}$ invariants (up to an overall multiplicative factor of $1 / 3$ ). By combining this result with those of the previous two sections, we arrive at a single generating function for the Gromov-Witten invariants of all $\Xi_{i}$.

\footnotetext{
${ }^{1}$ What happens is that for $w=0$, we get a prefactor of $\left.(3 p+\nu)(p+\nu)\right|_{p^{2}=0}=$ $4 p \nu+\nu^{2}$, while $w=1$ gives $\left.(3 p+\nu)^{2}\right|_{p^{2}=0}=6 p \nu+\nu^{2}$. This simply reflects the difference in the bundle structure of the two spaces.
} 


\subsection{A second approach to $\Xi_{2}$}

We provide here a second derivation of mirror symmetry for $\Xi_{2}$, which is along the lines of [6]. This calculation allows us to provide a closed form for the prepotential and mirror map of $\Xi_{2}$, similar to what was found in [6]. Note that we apply the cohomology relation $p^{2}=0$ to all toric vectors in this section.

The starting point is the (general) equivariant mirror symmetry calculation of $\mathcal{O}(1) \oplus \mathcal{O}(-3) \rightarrow \mathbb{P}^{1}$ with equivariant weights $(1,-\nu)$ acting on the bundle factors, which was carried out in [5]. Note that these are the most general equivariant Gromov-Witten invariants that can be computed for this space. This can be represented by charge vectors

$$
\left(\begin{array}{llll}
1 & 1 & 1 & -3 \\
& & 1 & -\nu
\end{array}\right)
$$

(Note that the 1 in the second row should strictly speaking be set to $\mu$, an equivariant parameter, but we have set this $\mu=1$ since the invariants computed using (3.43) are already the most general.) By mirror symmetry, we can use this to compute generalized equivariant Gromov-Witten invariants, and the potential turns out to be

$$
\begin{aligned}
W\left(\nu, x_{1}\right)= & x_{1}-\left(\frac{3}{4} \nu+\frac{3}{4} \nu^{2}+\frac{1}{4} \nu^{3}\right) x_{1}^{2} \\
& +\left(\frac{13}{12} \nu^{2}+\frac{9}{4} \nu^{3}+\frac{23}{12} \nu^{4}+\frac{3}{4} \nu^{5}+\frac{1}{9} \nu^{6}\right) x_{1}^{3}+\cdots
\end{aligned}
$$

Then $\nu=1$ gives physical Gromov-Witten invariants (i.e., the ones from the earlier section), while $\nu=-1$ gives the multiple cover formula (see [5]).

Now, one might initially hope that a clever choice of $\nu$ would allow us to match this to the two-curve prepotential (up to an overall factor of 2 ). Solving the obvious equation yields a value $\nu=12^{1 / 3}-1$, and after resubstituting this into (3.44), the result does turn out to be very, very close to the right answer. Unfortunately, there is an error term present that grows with increasing degree, and without the exact form of the error, the extraction of integer invariants is not possible. Nonetheless, this gives us a hint as to how to proceed: one might very well hope that some refinement of the theory (3.43) contains the looked-for invariants.

A quick glance at [6] reveals that there is indeed a perfectly natural refinement of (3.43), along the lines of the previous section. From [6], it was shown that the above $\nu$ dependent invariants can also be computed by using 
the charge vectors for the $D_{4}$ singularity

$$
\left(\begin{array}{lllll}
1 & 1 & 1 & -1 & -2 \\
& & 1 & -\nu & -\nu
\end{array}\right)
$$

which holds since the equivariant Euler classes of the bundles are the same, $-3 p-\nu=\left.(-2 p-\nu)(-p-\nu)\right|_{p^{2}=0}$. Then the most natural refinement of the equivariant theory defined by $(3.45)$ is of course

$$
\left(\begin{array}{ccccc}
1 & 1 & 1 & -1 & -2 \\
& & 1 & -\nu_{1} & -\nu_{2}
\end{array}\right)
$$

Then miraculously, with the simple specialization of weights $\nu_{1}=2, \nu_{2}=1$, we immediately recover the expansion for the $W_{\Xi_{2}}$ function.

Notice that this space also naturally contains mirror symmetry for $\Xi_{1}=$ $\mathcal{O}(1) \oplus \mathcal{O}(-3) \rightarrow \mathbb{P}^{1}$. This means we can simultaneously carry out mirror symmetry for $\Xi_{1}$ and $\Xi_{2}$. Namely, mirror symmetry for $\Xi_{1}$ is given by (here we have restored $\mu$, which was set to 1 in the above calculation)

$$
\left(\begin{array}{lllll}
1 & 1 & 1 & -1 & -2 \\
& & \mu & -\mu & -\mu
\end{array}\right)
$$

and hence mirror symmetry for $\Xi_{1}$ and $\Xi_{2}$ is determined by

$$
\left(\begin{array}{ccccc}
1 & 1 & 1 & -1 & -2 \\
& & \mu & -\mu-\nu & -\mu
\end{array}\right) .
$$

That is, $\nu=0$ gives $\Xi_{1}$ while $\nu=\mu$ is $\Xi_{2}$.

We note briefly that the $\Xi_{2}$ invariants can also be computed from the toric data

$$
\left(\begin{array}{cccccc}
1 & 1 & 1 & -1 & -1 & -1 \\
& & 1 & -2 \nu & -\nu & -\nu
\end{array}\right)
$$

which again follows since the equivariant Euler classes of both cases are the same. We will come back to this later in the paper.

\section{The localization calculation}

In this section, we reproduce the refined Gromov-Witten invariants of $K_{\mathbb{P}^{2}}$ derived in [1] by using the standard localization computation with specialized torus action weights on $\mathbb{P}^{2}$. The localization computation starts by 
assigning torus action weights to the homogeneous coordinates of $\mathbb{P}^{2}$ :

$$
\left(X_{1}: X_{2}: X_{3}\right) \mapsto\left(e^{\lambda_{1} t} X_{1}: e^{\lambda_{2} t} X_{2}: e^{\lambda_{3} t} X_{3}\right) .
$$

In the standard computation of local Gromov-Witten invariants of $K_{\mathbb{P}^{2}}$, we obtain the same results for an arbitrary choice of weights $\left(\lambda_{1}, \lambda_{2}, \lambda_{3}\right)$, but to obtain the refined invariants of [1], we specialize these weights as follows:

$$
\left(\lambda_{1}, \lambda_{2}, \lambda_{3}\right)=\left(1, \omega, \omega^{2}\right),
$$

where $\omega$ is the primitive cubic root of unity $-(1 / 2)+(\sqrt{-3} / 2)$. Of course, we can permute the subscripts of weights, and the following operations is also allowed:

$$
\left(1, \omega, \omega^{2}\right) \mapsto\left(e^{\lambda}, e^{\lambda} \omega, e^{\lambda} \omega^{2}\right),
$$

because these operations keep each amplitude $a(\Gamma)$, associated with the tree graph $\Gamma$ used in the localization computation of invariants, invariant. But any choice of weights which changes $a(\Gamma)$ is no longer allowed when computing refined invariants in [1]. The reason for this restriction comes from the following operation. The tree graph $\Gamma$ used in the localization computation of $K_{\mathbb{P}^{2}}$ consists of colored vertices $v(1), v(2), v(3)$ associated with homogeneous coordinates $X_{1}, X_{2}, X_{3}$ and of edges with degree $d$. Then the degree $d$ genus 0 local Gromov-Witten invariant of $K_{\mathbb{P}^{2}}$ is given by the following formula:

$$
\sum_{\Gamma, d(\Gamma)=d} a(\Gamma) q^{d(\Gamma)}
$$

where $q$ is the degree counting parameter and $d(\Gamma)$ is the degree of the graph $\Gamma$ defined by the sum of degrees of edges in $\Gamma$. But to obtain the refined invariants of $K_{\mathbb{P}^{2}}$ in [1], we have to introduce three different degree counting parameters $x_{1}, x_{2}, x_{3}$ that correspond to the three three $\mathbb{P}^{1} \mathrm{~s}$ in the toric diagram of $K_{\mathbb{P}^{2}}$. To realize this refinement, what we have to do is to define a colored degree $\left(d_{1}(\Gamma), d_{2}(\Gamma), d_{3}(\Gamma)\right)$ for the graph $\Gamma . d_{1}(\Gamma)$ (resp. $d_{2}(\Gamma)$, $\left.d_{3}(\Gamma)\right)$ is defined as the sum of degrees of edges whose boundary vertices consist of $v(2)$ and $v(3)$ (resp. $v(1)$ and $v(3), v(1)$ and $v(2)$ ). Then we change (4.4) as follows:

$$
\sum_{\Gamma, d(\Gamma)=d} a(\Gamma) x_{1}^{d_{1}(\Gamma)} x_{2}^{d_{2}(\Gamma)} x_{3}^{d_{3}(\Gamma)} .
$$


If we set the weights as proposed in (4.2), the above formula gives the generating function of refined invariants of [1] of total degree $d$. But if we set the weights in a way not allowed according to previous discussion, (4.5) turns out to be a meaningless polynomial. In the following, we write down the results of the computation in the form of a generating function up to total degree 6 :

$$
\begin{aligned}
& F(w):=\left(x_{3}+x_{1}+x_{2}\right) w \\
& +\left(-x_{1} x_{2}-\frac{7}{8} x_{3}^{2}-x_{2} x_{3}-\frac{7}{8} x_{2}^{2}-x_{3} x_{1}-\frac{7}{8} x_{1}^{2}\right) w^{2} \\
& +\left(3 x_{1} x_{3}^{2}+3 x_{2}{ }^{2} x_{3}+3 x_{2} x_{3}^{2}+3 x_{1} x_{2}{ }^{2}+\frac{55}{27} x_{1}{ }^{3}+\frac{55}{27} x_{3}{ }^{3}\right. \\
& \left.+3 x_{1} x_{2} x_{3}+3 x_{1}^{2} x_{3}+\frac{55}{27} x_{2}^{3}+3 x_{1}^{2} x_{2}\right) w^{3} \\
& +\left(-13 x_{1} x_{2}{ }^{3}-13 x_{1} x_{3}{ }^{3}-16 x_{1} x_{2} x_{3}{ }^{2}-\frac{121}{8} x_{1}{ }^{2} x_{3}{ }^{2}-\frac{455}{64} x_{2}{ }^{4}\right. \\
& -\frac{121}{8} x_{1}{ }^{2} x_{2}{ }^{2}-13 x_{1}{ }^{3} x_{2}-13 x_{2}{ }^{3} x_{3}-16 x_{1}{ }^{2} x_{2} x_{3}-16 x_{1} x_{2}{ }^{2} x_{3} \\
& \left.-13 x_{1}^{3} x_{3}-\frac{455}{64} x_{3}^{4}-\frac{121}{8} x_{2}^{2} x_{3}^{2}-\frac{455}{64} x_{1}^{4}-13 x_{2} x_{3}^{3}\right) w^{4} \\
& +\left(104 x_{1} x_{2} x_{3}{ }^{3}+68 x_{1}{ }^{4} x_{2}+91 x_{2}{ }^{3} x_{3}{ }^{2}+\frac{3876}{125} x_{2}{ }^{5}+91 x_{2}{ }^{2} x_{3}{ }^{3}\right. \\
& +68 x_{2}{ }^{4} x_{3}+112 x_{1} x_{2}{ }^{2} x_{3}{ }^{2}+68 x_{1} x_{3}{ }^{4}+112 x_{1}{ }^{2} x_{2} x_{3}{ }^{2}+91 x_{1}{ }^{2} x_{3}{ }^{3} \\
& +68 x_{1}{ }^{4} x_{3}+91 x_{1}{ }^{3} x_{2}{ }^{2}+112 x_{1}{ }^{2} x_{2}{ }^{2} x_{3}+91 x_{1}{ }^{2} x_{2}{ }^{3}+68 x_{2} x_{3}{ }^{4} \\
& +91 x_{1}^{3} x_{3}^{2}+104 x_{1} x_{2}^{3} x_{3}+104 x_{1}^{3} x_{2} x_{3}+68 x_{1} x_{2}^{4} \\
& \left.+\frac{3876}{125} x_{1}^{5}+\frac{3876}{125} x_{3}^{5}\right) w^{5} \\
& +\left(-399 x_{2}{ }^{5} x_{3}-\frac{4845}{8} x_{1}{ }^{2} x_{3}{ }^{4}-891 x_{1}{ }^{2} x_{2} x_{3}{ }^{3}-891 x_{1} x_{2}{ }^{3} x_{3}{ }^{2}\right. \\
& -891 x_{1} x_{2}{ }^{2} x_{3}{ }^{3}-741 x_{1} x_{2} x_{3}{ }^{4}-\frac{18496}{27} x_{1}{ }^{3} x_{3}{ }^{3}-\frac{4845}{8} x_{1}{ }^{4} x_{2}{ }^{2} \\
& -\frac{18496}{27} x_{1}{ }^{3} x_{2}{ }^{3}-\frac{4845}{8} x_{1}{ }^{2} x_{2}{ }^{4}-399 x_{1} x_{2}{ }^{5}-399 x_{1}{ }^{5} x_{2}-741 x_{1}{ }^{4} x_{2} x_{3} \\
& -891 x_{1}{ }^{3} x_{2} x_{3}{ }^{2}-\frac{33649}{216} x_{2}{ }^{6}-\frac{33649}{216} x_{3}{ }^{6}-\frac{33649}{216} x_{1}{ }^{6}-891 x_{1}{ }^{2} x_{2}{ }^{3} x_{3} \\
& -\frac{7533}{8} x_{1}{ }^{2} x_{2}{ }^{2} x_{3}{ }^{2}-891 x_{1}{ }^{3} x_{2}{ }^{2} x_{3}-\frac{4845}{8} x_{2}{ }^{4} x_{3}{ }^{2}-\frac{4845}{8} x_{2}{ }^{2} x_{3}{ }^{4}
\end{aligned}
$$




$$
\begin{aligned}
& -399 x_{2} x_{3}{ }^{5}-399 x_{1}{ }^{5} x_{3}-399 x_{1} x_{3}{ }^{5}-\frac{4845}{8} x_{1}{ }^{4} x_{3}{ }^{2} \\
& \left.-\frac{18496}{27} x_{2}{ }^{3} x_{3}{ }^{3}-741 x_{1} x_{2}{ }^{4} x_{3}\right) w^{6}+\cdots
\end{aligned}
$$

Note that the notation here differs from previous sections; above, $x_{i}$ are the equivariant parameters corresponding to the $\mu_{i}$ of Section 2 , and $w$ is the same as the $x_{1}$ of Section 3 . If we set $x_{3}=0$, we obtain generating function of local Gromov-Witten invariants of the two-curve cases with two Kähler parameters:

$$
\begin{aligned}
\left.F\right|_{x_{3}=0}(w):= & \left(x_{2}+x_{1}\right) w+\left(-\frac{7}{8} x_{2}{ }^{2}-x_{1} x_{2}-\frac{7}{8} x_{1}{ }^{2}\right) w^{2} \\
& +\left(\frac{55}{27} x_{1}{ }^{3}+\frac{55}{27} x_{2}{ }^{3}+3 x_{1} x_{2}{ }^{2}+3 x_{1}{ }^{2} x_{2}\right) w^{3} \\
& +\left(-13 x_{1} x_{2}{ }^{3}-\frac{455}{64} x_{1}^{4}-13 x_{1}{ }^{3} x_{2}-\frac{121}{8} x_{1}{ }^{2} x_{2}{ }^{2}-\frac{455}{64} x_{2}{ }^{4}\right) w^{4} \\
& +\left(68 x_{1} x_{2}{ }^{4}+\frac{3876}{125} x_{1}{ }^{5}+91 x_{1}{ }^{3} x_{2}{ }^{2}\right. \\
& \left.+\frac{3876}{125} x_{2}{ }^{5}+68 x_{1}{ }^{4} x_{2}+91 x_{1}{ }^{2} x_{2}{ }^{3}\right) w^{5} \\
& +\left(-399 x_{1} x_{2}{ }^{5}-\frac{18496}{27} x_{1}{ }^{3} x_{2}{ }^{3}-\frac{4845}{8} x_{1}{ }^{4} x_{2}{ }^{2}-\frac{33649}{216} x_{1}{ }^{6}\right. \\
& \left.-\frac{33649}{216} x_{2}{ }^{6}-\frac{4845}{8} x_{1}{ }^{2} x_{2}{ }^{4}-399 x_{1}{ }^{5} x_{2}\right) w^{6}+\cdots \\
\text { (4.7) } \quad &
\end{aligned}
$$

If we set $x_{1}=x_{2}=1, x_{3}=0$, we obtain one parameter generating function of the two-curve cases:

$$
\begin{array}{rl}
\left.F\right|_{x_{1}=x_{2}=1, x_{3}=0}(w):=2 & w-\frac{11}{4} w^{2}+\frac{272}{27} w^{3}-\frac{1771}{32} w^{4} \\
+ & \frac{47502}{125} w^{5}-\frac{81158}{27} w^{6}+\cdots
\end{array}
$$

We can easily see that $\left.w(d / d w) F\right|_{x_{1}=x_{2}=1, x_{3}=0}$ agrees with the result from the mirror symmetry computation. Moreover, if we set $x_{2}=x_{3}=0$, the 
result agrees with the one $\mathcal{O}(1) \oplus \mathcal{O}(-3)$ curve:

$$
\begin{aligned}
\left.F\right|_{x_{1}=1, x_{2}=x_{3}=0}(w):= & w-\frac{7}{8} w^{2}+\frac{55}{27} w^{3}-\frac{455}{64} w^{4} \\
& +\frac{3876}{125} w^{5}-\frac{33649}{216} w^{6}+\cdots
\end{aligned}
$$

\section{Revisiting and generalizing $\mathcal{O}(k) \oplus \mathcal{O}(-2-k) \rightarrow \mathbb{P}^{1}$}

\subsection{A new way to compute mirror symmetry for $\mathcal{O}(k) \oplus \mathcal{O}(-2-k) \rightarrow \mathbb{P}^{1}$}

The second main focus of this paper is to present an alternative formulation for the notion of mirror symmetry on $X_{k}=\mathcal{O}(k) \oplus \mathcal{O}(-2-k) \rightarrow \mathbb{P}^{1}$ proposed in [6]. This new viewpoint allows us to give a closed form for the mirror map for all $k$, and moreover produces the closed form for GromovWitten invariants found in [10]. This approach is closer to physical mirror symmetry than the formulation of [6], in the sense that we can use a single $I$ function for the computation for all $k$.

We first recall the work of [6]. The main observation of that paper was that the Gromov-Witten theory of $X_{k}$ with antidiagonal equivariant action $(\mu,-\mu)$ on the bundle is equivalent to Gromov-Witten theory on the space

$$
\mathcal{O}(1)^{\oplus k} \oplus \mathcal{O}(-1)^{\oplus(2+k)} \rightarrow \mathbb{P}^{1}
$$

with torus action

$$
(\underbrace{\mu \cdots \mu}_{k}, \underbrace{-\mu \cdots-\mu}_{2+k})
$$

on the bundle. The essential thing we needed to notice to see that these theories should be the same is that the equivariant Euler classes the respective bundles agree:

$$
\left.(k p+\mu)((-2-k) p-\mu)\right|_{p^{2}=0}=\left.(p+\mu)^{k}(-p-\mu)^{2+k}\right|_{p^{2}=0} .
$$

So, for example, consider $X_{2}=\mathcal{O}(2) \oplus \mathcal{O}(-4) \rightarrow \mathbb{P}^{1}$ with action $(\mu,-\mu)$ on the bundle. This can be represented torically as

$$
\left(\begin{array}{llll}
1 & 1 & 2 & -4 \\
& & \mu & -\mu
\end{array}\right),
$$

where the second row gives the equivariant weights acting on the bundle factors represented by $2,-4$. Then the results of [6] show that the same 
Gromov-Witten theory can be calculated by using the toric data

$$
\left(\begin{array}{llllllll}
1 & 1 & 1 & 1 & -1 & -1 & -1 & -1 \\
& & \mu & \mu & -\mu & -\mu & -\mu & -\mu
\end{array}\right)
$$

which corresponds to the space

$$
\mathcal{O}(1)_{\mu} \oplus \mathcal{O}(1)_{\mu} \oplus \mathcal{O}(-1)_{-\mu} \oplus \mathcal{O}(-1)_{-\mu} \oplus \mathcal{O}(-1)_{-\mu} \oplus \mathcal{O}(-1)_{-\mu} \rightarrow \mathbb{P}^{1}
$$

As usual, subscripts denote equivariant weights on the respective factors. The point of doing this reduction in [6] is that this allows us to find a very simple mirror map which agrees with [10], as well as to find a closed form for the Yukawa coupling.

Naturally, since only the agreement of equivariant Euler classes is needed for the above theories to coincide, the choice of bundle $\mathcal{O}(1)^{\oplus 2} \oplus \mathcal{O}(-1)^{\oplus 4}$ is not unique, so there may be any number of (possibly even simpler) theories which share the same Gromov-Witten invariants. One finds immediately that the equivariant Euler class of the bundle on $X_{k}$ is also the same on the space

$$
\left(\begin{array}{cccccc}
1 & 1 & 1 & -1 & -1 & -1 \\
& & \mu / k & -\mu / k & -\mu & -\mu
\end{array}\right) .
$$

To get some geometric insight into what this vector means, we note that the toric data of equivariant $\mathcal{O}(-1) \oplus \mathcal{O}(-1) \rightarrow \mathbb{P}^{1}$ with weights $(-\mu,-\mu)$ acting on the bundle is

$$
\left(\begin{array}{llll}
1 & 1 & -1 & -1 \\
& & -\mu & -\mu
\end{array}\right)
$$

and the difference between this and (5.6) is just the middle two columns, which represent the bundles $\mathcal{O}(1) \oplus \mathcal{O}(-1)$. Thus, this can be thought of as a 'twist' in the sense of Coates-Givental [4], which geometrically means that (5.6) is the total space

$$
\mathcal{O}(1)_{\mu / k} \oplus \mathcal{O}(-1)_{-\mu / k} \longrightarrow\left(\mathcal{O}(-1)_{-\mu} \oplus \mathcal{O}(-1)_{-\mu} \rightarrow \mathbb{P}^{1}\right)
$$

Hence, $\mathcal{O}(k) \oplus \mathcal{O}(-2-k) \rightarrow \mathbb{P}^{1}$ for all $k$ is just a twist of the $\mathcal{O}(-1) \oplus$ $\mathcal{O}(-1)$ curve.

Call the space defined by (5.6) $X_{k}^{\prime}$. Then, the advantage of working with $X_{k}^{\prime}$ rather than (5.1) is clear; the fact that the rank of the bundle of $X_{k}^{\prime}$ does not change (i.e., the bundle of (5.6) is rank 4 for all $k$, whereas (5.1) has 
rank $(2+2 k)$ allows us to use a single $I$ function to compute the answer for all $k$ (in contrast to (5.1)). To be completely explicit, the $I$ function corresponding to (5.1) for arbitrary $k$ is given as

$$
I_{1}=q^{p / \hbar} \sum_{d 0} \frac{\prod_{m=-d+1}^{0}(-p-\mu+m \hbar)^{2+k}}{\prod_{m=1}^{d}(p+\mu+m \hbar)^{k} \prod_{m=1}^{d}(p+m \hbar)} q^{d}
$$

while that for $(5.6)$ is

$$
I_{2}=q^{p / \hbar} \sum_{d \geq 0} \frac{\prod_{m=-d+1}^{0}(-p-\mu / k+m \hbar) \prod_{m=-d+1}^{0}(-p-\mu+m \hbar)^{2}}{\prod_{m=1}^{d}(p+\mu / k+m \hbar) \prod_{m=1}^{d}(p+m \hbar)} q^{d} .
$$

Then it is evident that $I_{2}$ can be Birkhoff factorized for arbitrary values of $k$, whereas $I_{1}$ cannot; thus we can use $I_{2}$ to find the general form of the mirror transformation for $X_{k}$.

Then, by going through the usual routine, from the above vector (5.6) we can expand in $\mu=\infty$ and perform Birkhoff factorization in order to extract the $J$ function for arbitrary values of $k$ :

$$
J_{k}=q^{p / \hbar}\left(1+\frac{\mu k(p(2+k)+1) \log (1+q)}{\hbar}+\frac{\tilde{W}_{k}(q)}{\hbar^{2}}+\cdots\right)
$$

i.e., the mirror map is

$$
t_{k}(q)=\log (q)+k(2+k) \log (1+q)
$$

in agreement with $[6,10]$. Then, after inverting the mirror map, we find

$$
\tilde{W}_{k}(q(t))=\mu(2 p k+2 p+\mu) W_{k}
$$

where remarkably, the coefficients of $W_{k}$ can easily be seen to have a very simple closed form:

$$
W_{k}=\sum_{j=1}^{\infty} \frac{(-1)^{(j-1) k}}{j^{2}(j-1) !} \prod_{m=1}^{j-1}(k(2+k) j+m) x^{k}
$$

where $x=e^{t}$. This is, of course, exactly the prepotential of [10], and, as we will see in the next section, a slight modification of this will allow us to deduce a closed form for the invariants of $\Xi_{2}$. 


\subsection{More general equivariant theories}

We now turn to a generalization of the above equivariant theory which, surprisingly, also turns out to have an elegant closed form, and further contains $\Xi_{2}$ invariants, which is how we derived the closed formula presented earlier.

The idea here is the following. From [5], mirror symmetry for $X_{1}$ can be performed by use of the vector

$$
\left(\begin{array}{llll}
1 & 1 & 1 & -3 \\
& & \mu & -\mu
\end{array}\right)
$$

Then, from [6], this can also be computed with

$$
\left(\begin{array}{llllll}
1 & 1 & 1 & -1 & -1 & -1 \\
& & \mu & -\mu & -\mu & -\mu
\end{array}\right) .
$$

Now, once we have uncovered the toric model (5.16), it is of course very natural to ask whether there might be some meaning to the equivariant Gromov-Witten theory of a generalized setup:

$$
\left(\begin{array}{cccccc}
1 & 1 & 1 & -1 & -1 & -1 \\
& & \nu_{1} & -\nu_{2} & -\nu_{3} & -\nu_{4}
\end{array}\right)
$$

Computationally speaking, this model simplifies dramatically if we specialize the weights to

$$
\left(\begin{array}{cccccc}
1 & 1 & 1 & -1 & -1 & -1 \\
& & \mu & -\mu & -\nu_{1} & -\nu_{2}
\end{array}\right) .
$$

The reason for this is, the third and fourth columns of (5.17) contribute the following factors to the $I$ function:

$$
\frac{\prod_{m=-d+1}^{0}\left(-p-\nu_{2}+m \hbar\right)}{\prod_{m=1}^{d}\left(p+\nu_{1}+m \hbar\right)} .
$$

Then, almost all the terms on the top and bottom will cancel if we set $\nu_{1}=\nu_{2}$ (that is, exactly one term on the top and one term on the bottom will survive). Hence, we consider instead the theories defined by (5.18).

To simplify the result, we take $\mu=1$. Let $J_{\nu}$ be the $J$ function obtained by Birkhoff factorization of (5.18). Amazingly, the result is almost the same 
as the previous section:

$$
J_{\nu}=q^{p / \hbar}\left(1+\frac{\left(p\left(\nu_{1} \nu_{2}+\nu_{1}+\nu_{2}\right)+\nu_{1} \nu_{2}\right) \log (1+q)}{\hbar}+\frac{\tilde{W}_{\nu}(q)}{\hbar^{2}}+\cdots\right)
$$

Moreover, we once again find a closed form for the Gromov-Witten invariants:

$$
W_{\nu}(x)=\sum_{k>0} \frac{(-1)^{k+1}}{k^{2}(k-1) !} \prod_{j=1}^{k-1}\left(k\left(\nu_{1} \nu_{2}+\nu_{1}+\nu_{2}\right)+j\right) x^{k}
$$

Here

$$
\tilde{W}_{\nu}(q(t))=\left(p\left(2 \nu_{1} \nu_{2}+\nu_{1}+\nu_{2}\right)+\nu_{1}+\nu_{2}\right) W_{\nu}(q(t)) .
$$

Now, from (5.18) we clearly see that

$$
W_{\left(\nu_{1}=1, \nu_{2}=1\right)}=W_{\Xi_{1}},
$$

i.e., the invariants agree with $\mathcal{O}(1) \oplus \mathcal{O}(-3) \rightarrow \mathbb{P}^{1}$, as they must. Also, as we saw above,

$$
W_{\left(\nu_{1}=1, \nu_{2}=2\right)}=W_{\Xi_{2}}
$$

which provides us with a closed formula for the Gromov-Witten invariants of $\Xi_{2}$.

\subsection{Connection open strings on $\mathbb{C}^{3}$}

We now outline the connection between closed strings on $\mathcal{O}(k) \oplus \mathcal{O}(-2-$ $k) \rightarrow \mathbb{P}^{1}$ and open strings on $\mathbb{C}^{3}$. Besides being of independent interest, we hope that eventually a generalization of this correspondence will allow us to define a two Kähler parameter toric model for $\Xi_{2}$.

We briefly recall the toric approach to open string theory given in [9]. Let $X$ be a non-compact Calabi-Yau 3-fold, which without loss of generality we take to have one Kähler modulus. As usual, we represent $X$ as a toric 
quotient

$$
X=\left\{z \in \mathbb{C}^{4}: \sum_{i} l_{i}\left|z_{i}\right|^{2}=r\right\} / S^{1}
$$

where

$$
S^{1}: z_{i} \longrightarrow e^{l_{i} \theta} z_{i}
$$

Clearly, the vector $l=\left(l_{i}\right)$ completely specifies $X$. Then, according to [9], open strings on $X$ with arbitrary framing $n$ can be represented by the vectors

$$
\left(\begin{array}{cccccc}
l_{1} & l_{2} & l_{3} & l_{4} & 0 & 0 \\
1 & -n-1 & n & 0 & 1 & -1
\end{array}\right)
$$

which is of course the toric data of a Calabi-Yau 4-fold. The idea behind this construction is that the second vector corresponds to the position of a Lagrangian submanifold at some point on the skeleton of the toric diagram of $X$, and this subsequently has an interpretation as a cycle in $\mathrm{M}$ theory.

Now, if one were to consider open strings on $\mathbb{C}^{3}$, since $\mathbb{C}^{3}$ has no Kähler modulus, the toric data of a single Lagrangian submanifold in $\mathbb{C}^{3}$ will be just

$$
\left(\begin{array}{lllll}
1 & -n-1 & n & 1 & -1
\end{array}\right) \text {. }
$$

The point is that if one looks at the toric diagram of this open string geometry (figure 3), it looks almost the same as the diagram of a $\mathcal{O}(k) \oplus \mathcal{O}(-2-$ $k) \rightarrow \mathbb{P}^{1}$ curve; this is the correspondence that was first observed at trivial framing in [2], and subsequently generalized to arbitrary framing in [10]. Then, it is a simple matter to use [6] to show that these theories are, in fact, exactly the same for arbitrary framing. All we have to do is to consider the equivariant version of the single-Lagrangian-in- $\mathbb{C}^{3}$ theory:

$$
\left(\begin{array}{ccccc}
1 & 1 & n & -n-1 & -1 \\
& & \mu & -\mu & -\mu
\end{array}\right)
$$

We then just have to compare this to the equivariant theory on $\mathcal{O}(k) \oplus$ $\mathcal{O}(-2-k) \rightarrow \mathbb{P}^{1}$ :

$$
\left(\begin{array}{cccc}
1 & 1 & k & -2-k \\
& & \mu & -\mu
\end{array}\right)
$$



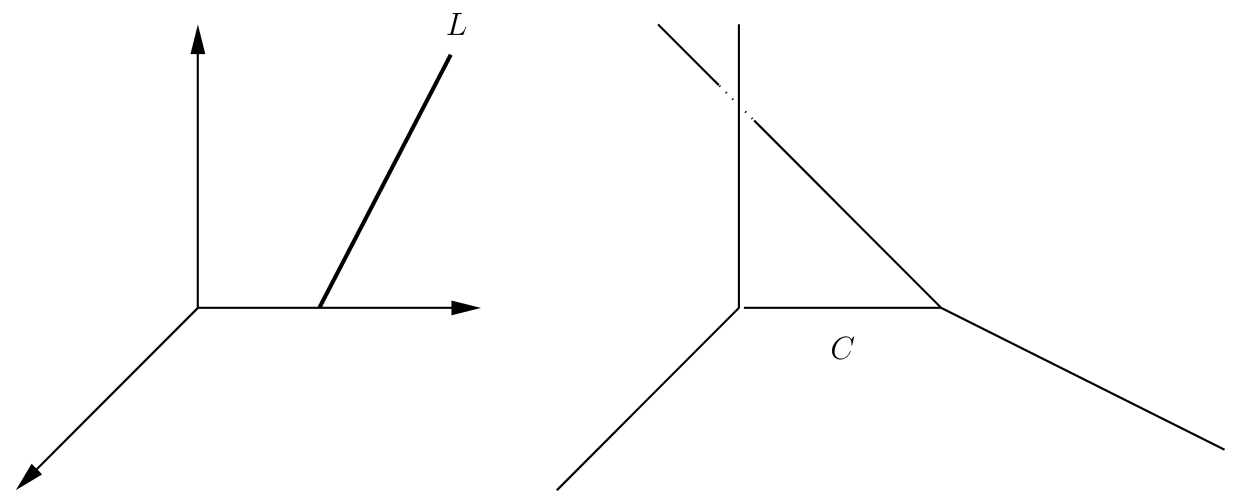

Figure 3: The comparison between $\mathbb{C}^{3}$ with one Lagrangian submanifold and a $\mathbb{P}^{1}$ with normal bundle $\mathcal{O}(k) \oplus \mathcal{O}(-2-k)$. Since the angle $L$ makes with the base of $\mathbb{C}^{3}$ is determined by the framing, we can think of the framing as determining the bundle structure of the right hand side picture. Note that the position of $L$ on the toric diagram of $\mathbb{C}^{3}$ maps to the Kähler parameter on $C$.

Then, as was shown in [6], the data of (5.30) are equivalent to that of

$$
\left(\begin{array}{ccccc}
1 & 1 & k & -k-1 & -1 \\
& & \mu & -\mu & -\mu
\end{array}\right),
$$

which is true simply because the equivariant Euler classes of the bundles of (5.30) and (5.31) are the same.

Then clearly, (5.29) and (5.31) agree, which shows quantitatively the correspondence between open strings on $\mathbb{C}^{3}$ with arbitrary framing and closed strings on $\mathcal{O}(k) \oplus \mathcal{O}(-2-k) \rightarrow \mathbb{P}^{1}$.

Finally, we mention that the two-curve problem $\Xi_{2}$, which consists of two curves with normal bundle $\mathcal{O}(1) \oplus \mathcal{O}(-3)$ intersecting at right angles, is supposed to be equivalent to the Gromov-Witten theory of open strings on $\mathbb{C}^{3}$ with two Lagrangian submanifolds on adjacent legs (figure 4) (M. Marino, private communication). Again, by looking at the toric diagram, we can see the formal similarity between these theories. However, it is not clear how one would define a toric model and Birkhoff factorization (which is required to compute the invariants in all the examples of this section) for a two parameter case. 

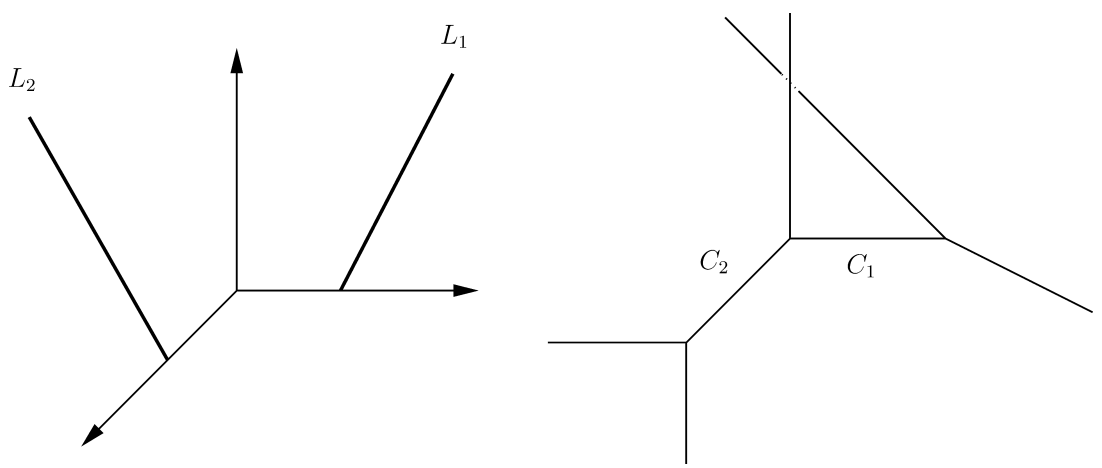

Figure 4: The comparison between two Lagrangian submanifolds in $\mathbb{C}^{3}$ and the case of two adjacent curves with arbitrary normal bundles (here, we have drawn $(-1,-1)$ and $(1,-3))$. Again, one can see a formal similarity between the two cases (for appropriate choices of framing on $L_{1}, L_{2}$ ).

\section{Conclusion}

In this paper, we have taken the first steps toward establishing a fully equivariant version of mirror symmetry for $K_{\mathbb{P}^{2}}$, by considering the special case a single independent equivariant parameter. We have also given a novel approach to localization which reproduces the fully equivariant invariants of [1]. This represents a major improvement over the results of [6], in that $K_{\mathbb{P}^{2}}$ has a non-trival 4 cycle, which, as we have seen, compounds the difficulty of the problem immensely.

Many questions are raised by our work here. The first and most obvious is how one could turn the contents of this paper into a complete theory of equivariant mirror symmetry of $K_{\mathbb{P}^{2}}$. The complication here is, as we have seen above, considering mirror symmetry for 'subgraphs' of the toric diagram for $K_{\mathbb{P}^{2}}$ requires Birkhoff factorization. At the same time, $K_{\mathbb{P}^{2}}$ 's mirror symmetry requires that we do not Birkhoff factorize. We have reconciled this problem in the present paper, but how this would generalize to a 3 equivariant parameter case is unclear.

Another troublesome issues are how to extend these techniques to a case with two Kähler parameters, such as $K_{F_{n}}$ for the Hirzebruch surfaces $F_{n}$, even for a single independent equivariant parameter. It is not immediately clear how this would work, since even for $K_{F_{1}}$, there is an $\mathcal{O}(1) \oplus \mathcal{O}(-3) \rightarrow \mathbb{P}^{1}$ curve in the geometry which should be visible in the fully equivariant model. We hope to address these questions in future work. 


\section{Appendix: Period integrals, Birkhoff factorization and mirror symmetry}

Here we collect the relevant background material on working out equivariant mirror symmetry from toric geometry. For simplicity, all considerations are restricted to the case of one Kähler parameter.

We can define a toric manifold $X$ by starting with a vector

$$
l=\left(\begin{array}{llll}
l_{1} & l_{2} & \cdots & l_{n}
\end{array}\right),
$$

where $l_{i}>0 \forall i$, and then using it to write a symplectic quotient

$$
X=\left\{\left(z_{i}\right) \in \mathbb{C}^{n}: \sum_{i} l_{i}\left|z_{i}\right|^{2}=r\right\} / S^{1}
$$

where the $S^{1}$ action is given as

$$
S^{1}:\left(z_{1} \cdots z_{n}\right) \rightarrow\left(e^{\sqrt{-1} l_{1} \theta} z_{1} \cdots e^{\sqrt{-1} l_{n} \theta} z_{n}\right)
$$

We can then consider the equivariant version of $X$ by including the torus action $X$ induced by that on $\mathbb{C}^{n}$ :

$$
\left(\mathbb{C}^{*}\right)^{n}:\left(z_{1} \cdots z_{n}\right) \rightarrow\left(\mu_{1} z_{1} \cdots \mu_{n} z_{n}\right)
$$

We represent this in this paper as a matrix

$$
\left(\begin{array}{lll}
l_{1} & \cdots & l_{n} \\
\mu_{1} & \cdots & \mu_{n}
\end{array}\right)
$$

This gives rise to the equivariant cohomology ring for $X$ :

$$
H_{T}^{*}(X, \mathbb{C})=\frac{\mathbb{C}[p] \otimes \mathbb{Q}[\mu]}{\left\langle\prod_{i}\left(l_{i} p+\mu_{i}\right)\right\rangle}
$$


From this data, we can construct Givental's equivariant $I$ function, which is supposed to give the period integrals of the mirror manifold to $X$ :

$$
I=q^{p / \hbar} \sum_{d \geq 0} \frac{1}{\prod_{i=1}^{n} \prod_{m=1}^{l_{i} d}\left(l_{i} p+\mu_{i}+m \hbar\right)} q^{d} .
$$

This is a series with coefficients taking values in $H_{T}^{*}(X, \mathbb{C})$.

We can then use [4] to consider mirror symmetry for the total space of $X$ with line bundles over it. Without loss of generality, we may restrict to the case $\mathcal{O}\left(k_{1}\right) \oplus \mathcal{O}\left(-k_{2}\right) \rightarrow X$ for $k_{1}, k_{2}>0$. Then from [4], the $I$ function is modified as follows:

$$
\begin{aligned}
I^{t w i s t}= & q^{p / \hbar} \sum_{d \geq 0} \frac{1}{\prod_{i=1}^{n} \prod_{m=1}^{l_{i} d}\left(l_{i} p+\mu_{i}+m \hbar\right)} \\
& \times \frac{\prod_{m=-k_{2} d+1}^{0}\left(-k_{2} p-\nu_{2}+m \hbar\right)}{\prod_{m=1}^{k_{1} d}\left(k_{1} p+\nu_{1}+m \hbar\right)} q^{d}
\end{aligned}
$$

and we are supposed to expand this twisted function about $\nu_{1}=\nu_{2}=\infty$.

In order to exhibit mirror symmetry, one must now use Birkhoff factorization, which goes as follows. Upon expansion of $I^{\text {twist }}$ in $\nu_{1}=\infty$, positive powers of $\hbar$ will be introduced into $I^{\text {twist }}$. We have to remove these via a procedure called 'Birkhoff factorization'. First, define the fundamental solution:

$$
S\left(\hbar, \hbar^{-1}\right)=M \times\left(\left.\left.I^{\mathrm{twist}}\right|_{p=-\mu_{1} / l_{1}} \quad \ldots \quad I^{\mathrm{twist}}\right|_{p=-\mu_{n} / l_{n}}\right)
$$

(here $M$ is a change of basis matrix which brings $S$ to the basis of solutions $\left.\left(\left.I^{\text {twist }}\right|_{p=0}, \ldots, d^{n} /\left.d p^{n} I^{\text {twist }}\right|_{p=0}\right)\right)$. Then this factors as

$$
S\left(\hbar, \hbar^{-1}\right)=Q^{-1}(\hbar) R\left(\hbar^{-1}\right) .
$$


Then $R\left(\hbar^{-1}\right)$ gives the 'factored' fundamental solution, from which we can write down the $J$ function, which takes on the form

$$
J=1+\frac{t(q)}{\hbar}+\frac{W(q)}{\hbar^{2}}+\cdots
$$

Then $t(q)$ is the mirror map, and we can recover the Gromov-Witten invariants of the configuration $\mathcal{O}\left(k_{1}\right) \oplus \mathcal{O}\left(-k_{2}\right) \rightarrow X$ from the function

$$
W(q(t)) .
$$

\section{Acknowledgments}

We owe special thanks to Marcos Marino for suggesting this problem, and for helpful correspondence. We would also like to thank C. Doran, S. Hosono, H. Iritani, B. Kim and K. Saito for helpful discussions.

\section{References}

[1] M. Aganagic, M. Marino and C. Vafa, All loop topological string amplitudes from Chern-Simons theory, Comm. Math. Phys. 247 (2004), 467-512, hep-th/0206164.

[2] M. Aganagic and C. Vafa, Mirror symmetry and a $G_{2}$ flop, JHEP 0305 (2003), 061, hep-th/0105225.

[3] J. Bryan and R. Pandharipande, The local Gromov-Witten theory of curves, arXiv:math.AG/0411037.

[4] T. Coates and A. Givental, Quantum Riemann-Roch, Lefschetz and Serre, arXiv:math.AG/0110142.

[5] B. Forbes and M. Jinzenji, J functions, non nef toric varieties and equivariant local mirror symmetry of curves, Int. J. Mod. Phys. A 22 (2007), 2327, arXiv:math.AG/0603728.

[6] B. Forbes and M. Jinzenji, Local mirror symmetry of curves: Yukawa couplings and genus 1, Adv. Theor. Math. Phys. 11 (1) (2007), 175-197, arXiv:math/0609016.

[7] H. Iritani, Quantum D-modules and generalized mirror transformations, Math. Z. 252(3) (2006), 577-622, arXiv:math/0411111. 
[8] M. Jinzenji, Coordinate change of Gauss-Manin system and generalized mirror transformation, Int. J. Mod. Phys. A 20(10) (2005), 2131-2156.

[9] W. Lerche, P. Mayr and N. Warner, $N=1$ special geometry, mixed hodge variations and toric geometry, hep-th/0208039.

[10] N. Caporaso, L. Griguolo, M. Marino, S. Pasquetti and D. Seminara, Phase transitions, double-scaling limit, and topological strings, hepth/0606120.

Research Institute for Mathematical Sciences

KYOTO UNIVERSITY

КҮото 606-8502

JAPAN

E-mail address: brian@kurims.kyoto-u.ac.jp

DiVISION OF MATHEMATICS

Graduate School of Science

HOKKAIDO UNIVERSITY

SAPPORO 060-0810

JAPAN

E-mail address: jin@math.sci.hokudai.ac.jp

Received October 4, 2007; Accepted November 27, 2007 